\title{
Bilingualism and Speech Sound Disorders
}

\author{
Brian A. Goldstein ${ }^{1}$ - Christina E. Gildersleeve-Neumann ${ }^{2}$
}

Published online: 23 June 2015

(C) Springer International Publishing Switzerland 2015

\begin{abstract}
The nature of speech sound development and disorders in bilinguals is complex, in part, because of the interdependence between languages. That interdependence, however, appears to be advantageous to bilinguals in that it bootstraps the languages such that speech sound development is similar, although not identical, to that of monolinguals. The same principle also applies to bilinguals with speech sound disorders. From a longitudinal perspective, speech sound acquisition in typically developing bilinguals and those with speech sound disorders is not remarkably different than that of monolinguals. However, speech sound skills are neither identical across the languages nor equally distributed across the languages given each language's phonotactic constraints. Thus, assessing speech sound skills in bilinguals is more complex than that for monolinguals. Typically, standardized assessments are not available for differential diagnosis, and informal measures are required. Intervention for bilinguals with speech sound disorders likely will need to occur in all languages.
\end{abstract}

Keywords Bilingualism · Speech · Articulation · Phonology $\cdot$ Development $\cdot$ Spanish $\cdot$ Intervention $\cdot$ Best practices $\cdot$ Assessment $\cdot$ Disorder

This article is part of the Topical Collection on Specific Language Impairment/Speech Sound Disorder

Brian A. Goldstein

goldstein@lasalle.edu

Christina E. Gildersleeve-Neumann

cegn@pdx.edu

1 Department of Communication Sciences and Disorders, La Salle University, 1900 W. Olney Av, Philadelphia, PA 19141, USA

2 Speech and Hearing Sciences Department, Portland State University, PortlandPO Box 751OR 97207, USA

\section{Introduction}

The majority of the world's population is bilingual [1]. Individuals who are acquiring more than one language are known by many different labels: bilinguals, dual language learners, multilinguals, etc. Those static terms obscure the fact that bilingualism lies on a continuum [2]. That is, the language skills of bilinguals can vary from relatively equal use and proficiency in both languages to high levels of use and proficiency in one language and relatively lower levels of use and proficiency in the other language(s). Differences in use and proficiency along that continuum have effects across all domains of language including phonology. Those differences in the phonological domain have important and significant implications for bilinguals with speech sound disorders (SSD).

\section{Theories of Phonological Development and SSD}

Two commonly espoused theories of bilingual language acquisition are the Unitary System Model and the (Interactional) Dual Systems Model [3]. According to the Unitary System Model, linguistic skills initially are not separated by language by the child. Through time and experience, children learn to separate the two systems. According to the (Interactional) Dual Systems Model, the more widely accepted theory, linguistics skills are separated early in development, although there is interaction between the two languages such that children leverage skills in one language to assist in the development of skills in the other language $[4 \bullet, 5 \bullet]$. One weakness of this theory is that the nature of the interdependence is underspecified. Thus, theories such as Dynamic Systems Theory (DST), which serves to account for the behavior of complex systems, have been applied to language acquisition. DST focuses on how seemingly autonomous units are 
interconnected during development, being studied in depth in motor learning but more recently applied to language acquisition of monolinguals [6], bilinguals, and to those with SSD. For example, Gildersleeve-Neumann and colleagues explored the speech development of 7 Quichua learning 9- to 16-month olds and found that many production factors accounted for the infants' early vocalizations [7]. Moreover, perception and production dynamically interacted to account for differences in the production of babbling and early words [8]. Rvachew and Bernhardt treated 48 children with SSD and randomly assigned them to two groups based on the complexity of their treatment targets [9]. Their findings indicated that dynamic interactions across subcomponents, such as articulatoryphonetic knowledge, a pre-existing phonological system, and new phonological knowledge resulted in the emergence of more complex forms through the treatment of less complex forms.

DST also has been applied to language development in bilinguals, with progression and regression characteristics observed [10]. Phonological skills will increase and decrease over time as dictated by a number of variables such as environment, language history, language use, proficiency, and phonemic and phonetic factors affecting that development. From a DST perspective, developmental progression may include the appearance of surface-level regression due to underlying reorganization and progression in the developing system [11]. Evidence for progression of phonological skills comes from studies examining development by bilinguals in comparison to monolinguals. Results from those studies indicate that phonological development and skills in bilingual children are not significantly different from those in monolingual children [4, 12]. Studies have noted, however, slight regression in the phonological skills of some typically developing bilinguals in comparison to monolinguals $[12,13]$ and in those with SSD [14]. Gildersleeve-Neumann, Peña, Davis, and Kester found surface-level regression in Spanish phonological skills 9 months after the introduction of English to five Spanish-English pre-schoolers, suggesting overall development as these sequential bilingual children shifted from monolingual to bilingual phonological systems [15•]. Finally, Gildersleeve-Neumann and Goldstein found evidence supporting assumptions made in DST in the treatment of two Spanish-English speaking children with SSD [16•]. They found that treating SSD in both languages resulted in positive changes in both languages, some non-treated errors, and overall speech accuracy.

\section{Typical Speech Sound Development}

Speech sound (both segment and pattern based) development in bilingual children is often thought to be slower than that of monolingual children. Data from a number of studies seem to indicate otherwise, especially if a longitudinal view of acquisition is considered. The data are even more compelling if commensurate skills in bilinguals compared to monolinguals are considered. That is, research on typical speech development in bilingual children indicates phonological skills which are less advanced, more advanced, and commensurate to those of monolingual children.

Research findings from a number of studies have indicated that the speech sound skills of bilinguals are less advanced than those of monolinguals. For example, in examining the phonological skills of 16 Cantonese-English speaking children, Dodd, So, and Wei found that their skills were less advanced than those of monolingual peers [17]. The comparisons across the two groups, however, were retrospective in nature. In a group of Spanish-English-speaking 3-year olds, Gildersleeve-Neumann, Kester, Davis, and Peña found that, in English, the bilingual children showed an overall lower intelligibility rating, made more consonant and vowel errors overall, and produced more uncommon error patterns, some of which were the result of cross-linguistic effects, than sameaged monolingual English peers [13]. Despite the less advanced skills of the bilingual children compared to their monolingual counterparts, the speech sound skills of the bilingual children were within normal limits [4•]. That is, they were not at-risk for being identified with a speech sound disorder simply because they were acquiring more than one language. Finally, in a group of 12 4-year-old Spanish-Englishspeaking children, Goldstein and Washington found that the bilingual children were significantly less accurate than monolingual children on three sound classes in Spanish (spirants, flap/tap, and trill in Spanish) [12].

Speech sound skills of bilingual children have been shown to be more advanced than their monolingual peers. In a group of 241 Maltese-English-speaking bilingual children, Grech and Dodd found that the bilingual children showed higher consonant accuracy and fewer error patterns in comparison to monolinguals [18]. Kehoe, Trujillo, and Lleó and Lleó, Kuchenbrandt, Kehoe, and Trujillo found a higher rate of coda production in the Spanish productions of three SpanishGerman-speaking bilinguals than in those of three monolingual Spanish speakers [19, 20].

Numerous studies examining speech sound skills in bilinguals have shown that their skills are commensurate with those of monolingual peers. Such results have been seen in Spanish-English bilingual children [4 , 12, 21] and in Russian-English bilingual children [22]. Commensurate skills across the bilingual and monolingual groups have extended to overall consonant accuracy, accuracy on sound classes, wordshape complexity, phonetic inventories, and percentages of occurrence for phonological patterns. Overall, findings from these studies showed that bilingual children generally exhibited a similar rate of acquisition compared to monolinguals 
and that their speech sound skills were within normal limits compared to monolingual peers.

\section{Bilinguals with SSD}

Relative to the number of studies on speech sound development in typically developing bilingual children, those focusing on bilingual children with speech sound disorders are few. Existing studies indicate that the phonological skills of bilingual children with speech sound disorders are both aligned with and different from those of monolingual speakers with speech sound disorders. For example, both bilingual and monolingual children with speech sound disorders exhibit errors on many of the same types of elements: consonant clusters, multisyllabic words, and liquids [14]. Moreover, research suggests that bilingual and monolingual children with speech sound disorders do not differ significantly on place of articulation and manner of articulation categories and on measures of consonant accuracy and complexity [23]. The bilingual children, however, did demonstrate higher percentages of occurrence on some phonological patterns in comparison to monolingual speakers [14]. Finally, as was the case with monolingual speakers, phonological skills are distributed across the two languages. For example, in a group of 18 Spanish-English bilingual children with speech sound disorders ranging from 3.3 to 5.8 (mean $=45$ months), Goldstein, Bunta, Lange, Burrows, Pont, and Bennett found significant differences between languages for accuracy on vowels, two manner classes (stops and nasals), early- and late-developing sounds, and percentages of occurrence for four phonological patterns (stopping, gliding, final consonant devoicing, and fronting) [24].

The results of these studies indicated that bilingual speakers with speech sound disorders showed similar phonological skills to their monolingual counterparts. That said, differences across the groups did exist for a subset of phonological skills. Finally, phonological skills for such children are not similarly distributed across the two languages.

\section{Variables to Consider}

One hallmark of bilingual language acquisition is crosslinguistic effects (i.e., the influence of one linguistic system on another [25]). Such influences come from contact between the two languages based on a speaker's motivation, a speaker's oral and perceptual abilities, and the prestige of the various dialects [26]. In terms of phonology, cross-linguistic effects extend to both segmental and suprasegmental properties. For example, a Hmong-English bilingual child might use an aspirated affricate [ ] in an English production because that sound is part of the Hmong phonetic inventory. In general, it seems that the frequency of cross-linguistic effects is relatively low [4•], but significant variation has been noted in younger children [27]. Such wide variation in linguistic skills across bilingual speakers is not uncommon, however. What these studies do indicate is the interdependence across the two languages. Even though children largely keep the two languages separate, there is interaction between the two systems.

One influencing factor on the use of cross-linguistic effects might be the effect of language experience on the development of phonological skills. Such experience is typically put in the context of the dominance of one language over the other. Findings from studies examining the effect of language dominance on phonological skills are equivocal. For example, Paradis found an effect in the production of syllable omissions in French-English-speaking bilinguals [3], but Law and So did not find such an effect in the acquisition of Cantonese and Putonghua phonology in a group of simultaneous bilingual Cantonese- and Putonghua-speaking children [28]. Examining dominance more finely, Goldstein, Bunta, Lange, Rodriguez, and Burrows found that estimates of parentreported output (i.e., the percentage of time the children spoke each language) did not significantly predict segmental accuracy [29]. However, estimates of parent-reported language use (i.e., how often the children used each language) and language proficiency (i.e., how well the children used each language) did predict segmental accuracy. These effects differed by language, again showing the distributed nature of phonological skills across the two languages.

\section{Assessment}

The earlier a child's speech sound disorder is identified, the earlier that child can receive supports to improve their speech and communication. Identification of speech sound disorders requires measurement that is valid and efficient, both for monolingual and bilingual children. Assessment of children's speech sound development is typically conducted with standardized single-word picture naming tasks. Connected speech sampling can also be used to provide information on intelligibility and how sounds are used spontaneously and in longer utterances. Standardized tests provide a relatively quick and efficient way to understand a child's speech since they are designed to include productions of the language's phonemes in multiple-word positions. Standardized norm-referenced tests allow comparison of the child's performance to a large sample of their peers and to determine whether a child's speech is within a typical range for development. While standardized normreferenced tests have been the ideal for the purpose of differential diagnosis, they are most accurate for use with a homogenous population: typically monolingual children from a single dialectal background. Standardized scores do 
not validly differentiate typical from atypical speech in children who are not adequately represented in the norming population.

Assumptions of homogeneous language environments do not fit the heterogeneous language environments of bilingual children's experiences. As discussed, the language environment of every bilingual child is unique. Bilingual children differ across languages in proficiency, use, dialect, communication partners, communication environments, and the relative status of each of the child's language in the eyes of the child and of the community. For bilingual children, a standardized norm-referenced test that can address the individual needs of all bilingual children and take into account these variables may not be possible nor cost-effective, since the norming population for all individual bilingual children will be difficult to define. The phonological subtest of the Bilingual English-Spanish Assessment (BESA) is an assessment tool normed on 1112 bilingual children to assess the speech and language abilities of English-Spanish children ages 4 through 6 years. Although the BESA is a promising assessment for some bilingual children, to create a test that accounts for each bilingual child's unique language factors with a large enough norming population to provide valid standardized scores seems unlikely and cost-prohibitive. It is likely that the most valid assessment of many bilingual children will include a systematized and individualized measurement and not necessarily a standardized norm-referenced assessment of speech.

The valid assessment of a bilingual child requires understanding of his or her entire speech system. This understanding cannot be achieved by testing only one of a bilingual child's languages, regardless of whether the language tested appears dominant. To fully understand intelligibility, strengths, and weaknesses, a bilingual child's speech sound production must be assessed in all languages. To do so, the following information must be collected. The assessment must determine which sounds the child produces in each language (the phonetic inventory), which sounds are meaningfully contrasted in each language (the phonemic inventory), the types of errors the child makes, and if those errors occur in patterns. Assessment of prosodic information (for example, word- and phrase-level stress patterns, intonation, loudness) should also be included. Information on consistency of errors is useful for differentiating type of speech sound disorder (e.g., functional versus childhood apraxia of speech [30, 31]). Percent consonant correct is important for determining severity of the speech sound disorder [32]. Since vowels often differ more than consonants across languages, exploring vowel accuracy and types of vowel errors will shed light on how much of the vowel error carryover is cross-linguistic in nature and whether the percent error is typical for a bilingual child $[13,15 \bullet]$. Understanding inventory, error patterns, and accuracy is important for all languages and will require assessment in each language separately. This language-specific information can then be compared to see how different errors affect each language and if the frequency differs across languages.

In addition to assessing the bilingual child's languages separately, a composite phonological assessment of the bilingual child is prudent. A composite assessment allows the speechlanguage pathologist (SLP) to determine if the child's phonetic inventory differs across languages, if error rates differ across languages, and if a child is producing a phoneme in one language but not the other, whether one language has a larger inventory or differing error rate than the second. This whole phonological system view of the child should be compared to individual performance in each language. These findings will likely provide helpful information in designing and implementing intervention for the child-knowing whether some information can be transferred from one language to the other, for example.

Ideally, SLPs who assesses a bilingual child should have a similar language background to the child they are assessing, speaking both languages and even the same dialects with the child. This shared language experience will likely result in the SLP having the fullest understanding of the bilingual phonological system of the child-where there are deficiencies, where cross-linguistic effects occur, and what strengths and weaknesses the child has in each language. In practice, however, most SLPs will not share the bilingual language environment of all children they are assessing. Unfortunately, understanding only one language provides only a partial picture of a child's overall phonological system. In these cases, the SLP still needs to ensure a thorough assessment of each of the child's languages. While the SLP may not speak all of the child's languages and will need to rely on an interpreter for direct assessment and interpretation of the assessment in the other language(s), the SLP still has the responsibility for the overall assessment of the child. This requires that the SLP understand what the child's sound system looks like — overall and in each language. The SLP who does not speak one or more of the child's languages needs to research characteristics of the second language, having an awareness of phonemes in both languages and their phonotactic rules. A full understanding of dialectal information is important as well. The SLP needs to consider how the phonology of the child's unknown language differs from the shared language, and how these differences may affect speech production in both languages. While the SLP will not be able to directly conduct the assessment in the non-shared language (direct assessment would not be authentic since it would not be a meaningful communication endeavor), the SLP will need to work with an interpreter in the best assessment of the unknown language, informing the interpreter of the sounds you are looking for, making sure the interpreter is familiar with the dialectal properties of the 
non-shared language phonological system, requesting that the interpreter looks for cross-linguistic effects in the non-shared language.

There are promising new directions for the assessment of bilingual children. Parent report is being used to assess children's early language development $[33,34]$ and as an assessment measure in language disorders in bilingual children [35, 36]. There is also preliminary research suggesting a high correlation between parent report on speech and accuracy measures of percent consonants correct (PCC) and percent vowels correct (PVC) in a sample of 24 Spanish monolingual children [37]. Follow-up research with 263 children has shown that parent's responses on 6 of the 10 original questions differentiate children with typical from atypical speech development with $94 \%$ specificity and $85 \%$ sensitivity [38]. While these findings are preliminary, future exploration of parent report as an efficient and effective assessment tool is warranted.

Another promising area for valid speech assessment of bilingual children is the use of dynamic assessment measures. Static assessments, such as single-word naming tasks, use a standard score to differentiate typical from atypical development in children. However, static assessment only tells the examiner that a child did not produce a word correctly but does not provide information as to why the error occurred. Thus, it is difficult to know if a child's errors are indicators of atypical speech development, unfamiliarity with the speech tasks, or typical for a child from their bilingual language background. In contrast to static assessment measures, dynamic assessments evaluate the potential developmental level that the child receives when assisted by the examiner [39]. Potential development is assessed by changing the production environment (phonetic context, word length, etc.) as well as by supporting production through cueing strategies, allowing the examiner to determine how stimulable the child is for speech sounds or word shapes that are in error.

\section{Intervention}

It is clear that bilingual children with speech sound disorders need intervention to improve speech skills and overall communication; however, research has not determined if there is a best approach to choosing the language(s) for intervention for bilingual children. As discussed, many current theories of bilingual speech development predict some level of interaction between the child's languages; therefore, it seems that treatment that facilitates speech development in all languages is likely to have the largest overall impact on the child's communication. However, there is extremely limited research on intervention for bilingual children with speech sound disorders. All of these research studies have been done on bilingual children in the USA and on children whose second language is English. Many studies have been case studies, with little rigor in their design and difficulty in interpreting whether the information applies to other individuals. Also, many of the studies have only provided pre- and post-comparisons of speech skills in one language without a controlled research design. In a pre-/post-case study design, Holm, Ozanne, and Dodd reported on the English treatment of SSD for a 5-year-old Cantonese-English-speaking boy who heard and spoke Cantonese at home until the age of 3.3 [40]. The child's two-phase treatment targeted distorted /s/ and then cluster reduction. Findings showed that English articulatory treatment $/ \mathrm{s} /$ resulted in improvement in $/ \mathrm{s} /$ in both languages; however, the treatment of cluster reduction only improved cluster production in English and not in Cantonese. However, Cantonese is not viewed as containing clusters in its phonology, and thus, the word shape cross-language effects are difficult to interpret. Holm and Dodd, using another pre-/post-case study design, examined cross-linguistic generalization in two children, one Cantonese-English and one Punjabi-English [41]. Both children were first exposed to English at age 3. In the CantoneseEnglish child, 7 weeks of articulatory treatment for distorted / $\mathrm{s} /$ in English resulted in improvements in English on the targeted sound and a few sounds that were not directly targeted: / // and /s/ clusters. Generalization was observed on Cantonese on the phonemes $/ \mathrm{s} /$ and $/ \mathrm{ts} /$. Subsequent treatment for cluster reduction in English did not show generalization across the children's other languages. Ray used a cognitivelinguistic treatment approach with a 5-year-old trilingual (Hindi, Gujarati, and English) boy with a mild SSD [42]. The child, born in the USA, was first exposed to English at age 4 although he had had exposure to English prior. Minimal contrast therapy in English was used to target multiple error patterns (final consonant deletion, gliding, cluster reduction). Results indicated that treatment was effective in decreasing the percentage of occurrence of error patterns and increasing consonant accuracy in all three languages and improving the child's percentile rank on a standardized English articulation assessment. Although phonological skills were examined in all languages, the effect of clinician and home practice was not accounted for, nor was it clear if and how generalization took place across the child's languages. These three studies of intervention for bilingual children with SSDs have not shown that monolingual English approaches are more effective than bilingual approaches, nor have they determined whether cross-linguistic generalization would be more likely to occur if the treatment were bilingual or in the children's home languages.

Recently, Gildersleeve-Neumann and Goldstein explored bilingual intervention for speech sound disorder using a single subject multiple baselines across behavior experimental design [16•]. Two 5-year-old sequential Spanish-English boys received bilingual intervention for 8 weeks. Both boys were stronger in Spanish than English. Their speech skills were compared pre- and post-treatment, and ongoing treatment 
performance was measured throughout treatment in the form of ongoing probes. The speech of both children improved in both languages, both on treated errors and on overall speech accuracy measures, suggesting that a bilingual treatment approach was effective. Interestingly, both boys received more Spanish than English treatment, yet their English speech skills improved as much as their Spanish, perhaps suggesting that treatment in the stronger language could be more efficient for treating the child's non-dominant language.

There is clearly a need for treatment research related to language of intervention. Nevertheless, it is safe to assume that since bilingual children with speech sound disorders will manifest their speech sound disorder in both languages, it is important that both languages be considered and likely receive intervention at some point throughout the course of the treatment. How each language is treated may differ depending on the child and the situation, with some children with SSD needing direct intervention in both languages throughout and others primarily receiving direct intervention in one language with the other language(s) treated through brief carryover activities or homework assignments.

Bilingual children with a speech sound disorder have difficulty producing words and phrases in both languages spoken in their environment. As noted, the speech sound disorder of a bilingual child will be present in both of their languages. The child internal factor that results in a speech sound disorderbe it deficits in articulatory, phonological, motor planning, or a combination of these - will determine the type of speech treatment needed. For example, deficits in motor planning warrant treatment approaches that teach sound production, support correct sound production, and support practice of sound production. A variety of treatment approaches have been shown effective for children with speech sound disorders. There is every reason to believe that these treatment approaches will be effective for bilingual children with SSD as well. The language environment (monolingual or bilingual) of the intervention and how that environment changes over time must be carefully considered to address the unique needs of the bilingual child so that intervention is maximized.

In addition to treating the child internal factor that is resulting in the speech sound disorder, a bilingual child needs intervention that facilitates growth in speech production capabilities in the languages the child communicates in. To best treat bilingual children with a speech sound disorder, we must determine which language environment is optimal for the bilingual treatment, as well as which language environment(s) are effective.

Because no large-scale controlled intervention research exists, it is difficult to use evidence-based approaches to determine the best treatment approach for bilingual children. In the absence of such evidence, expert opinion and clinician-based factors will have to inform decisions on treatment approach. Ebert and Kohnert suggest that a number of factors affect treatment success and many of these factors suggest how critical a bilingual environment for intervention will be [43]. These factors include clinician's rapport with client, the functionality of the treatment for the child, how well the clinician communicates with the client, the extent of communication the clinician has with the family, and the clinician's willingness to adapt their treatment objectives and the activities to meet the client's needs. All of the factors, critical for treatment success, emphasize the importance of a bilingual approach for a bilingual child, in particular if the child's family language(s) are not those of the academic environment.

Important intervention considerations regardless of language environment are (1) choosing the treatment goals based on specific speech needs (increasing phonetic inventory, decreasing segmental, or syllabic- or word-level error patterns), (2) choosing specific targets to achieve treatment goals (specific sounds to increase the child's inventory, for example), and (3) choosing how to target the goals. This could include targeting one goal at a time (vertical attack strategy), more than one goal at a time (horizontal strategy), or cycling through goals.

After determining intervention needs specific to the child's type of speech sound disorder, the bilingual child's unique language needs must be addressed. These include determining whether to use a bilingual (treatment of the goals in both languages) or a cross-linguistic (treat goals specific to a single language) approach [14]. It is likely that the same child will have need for both bilingual and cross-linguistic approaches at different times in the course of the intervention, with bilingual approaches critical for shared phonemes, word shapes, and prosodic structures, and cross-linguistic approaches needed for unique components of each language's speech system. In addition to determining the need for bilingual or crosslinguistic approaches, the therapist must consider how to design therapy to meet the dual language goals: should both languages be treated in the same session? Or should language of intervention alternate across sessions? Or should the overall intervention plan focus on one of the child's languages, with a structured approach to support cross-language transfer to the child's other language(s), such as meta-phonological strategies at the end of each session that clarify how the treatment conducted in one language applies to the child's other language(s).

Finally, intervention for the child with the speech sound disorder must include ongoing monitoring of progress in each language, ensuring that the child's intelligibility and accuracy is increasing in all communication situations. Probe data can be collected in each language to monitor progress. If probe data are only collected in one language, it is impossible to know whether overall progress is occurring in the speech skills of the bilingual child, or whether improvement in one language is coming at the expense of decreases in speech skills in the child's other language(s), thereby resulting in no 
overall improvement in the child's bilingual phonological system.

\section{Conclusions}

There is a growing body of research exploring bilingual speech development and disorder. This research demonstrates how bilingual children share similar developmental milestones with monolingual children and how bilingual children differ from monolinguals in the ways in which they become proficient in each language they use and in the overall development of a bilingual phonological system. While the research evidence for best assessment and intervention practices for bilingual children with speech sound disorders is limited, small-scale studies and expert opinion indicate the need for a whole-child perspective, including assessment of the bilingual child in all of their languages. Growing evidence also suggests the benefits of a bilingual approach to intervention even when the SLP does not share all of the child's languages. Continued research on all of these topics is greatly needed.

\section{Compliance with Ethics Guidelines}

Conflict of Interest Brian A. Goldstein receives a royalty from the Bilingual English Spanish Assessment. He has no other financial or nonfinancial conflicts of interest.

Christina Gildersleeve-Neumann declares that she has no conflict of interest.

Human and Animal Rights and Informed Consent This article does not contain any studies with human or animal subjects performed by any of the authors.

\section{References}

Papers of particular interest, published recently, have been highlighted as:

- Of importance

1. Grosjean F. The extent of bilingualism. In: Grosjean F, editor. Bilingual: life and reality. Cambridge, MA: Harvard University Press; 2010.

2. Valdés G, Figueroa RA. Bilingualism and testing: a special case of bias. Norwood, NJ: Ablex; 1994.

3. Paradis J. Do bilingual two-year-olds have separate phonological systems? Int J Biling. 2001;5:19-38.

4. Fabiano-Smith L, Goldstein B. Phonological acquisition in bilingual Spanish-English speaking children. J Speech-Lang Hear Res. 2010;53:160-78. This study indicates that transfer, deceleration, and a variation of the acceleration hypothesis occur in bilingual phonological acquisition. Results indicated separation and interaction between the two languages of the bilingual children.
5. Paradis J, Genesee F. Syntactic acquisition in bilingual children: autonomous or interdependent? Stud Second Lang Acquis. 1996;18:1-25. Results from this study indicated no evidence for transfer, acceleration, or delay in syntactic acquisition and showed evidence for autonomous grammar acquisition.

6. Thelen E, Bates E. Connectionism and dynamic systems: are they really different? Dev Sci. 2003;6:378-91.

7. Gildersleeve-Neumann CE, Davis BL, MacNeilage PF. Syllabic patterns in the early vocalizations of Quichua children. Appl Psycholinguist. 2013;34:111-34.

8. Gildersleeve-Neumann, C. E. Constraints on infant speech acquisition: a cross-linguistic perspective. (Doctoral Dissertation). Retrieved from ProQuest Dissertation and Theses Accession Order No: [3032398] 2001.

9. Rvachew S, Bernhardt BM. Clinical implications of dynamic systems theory for phonological development. Am J Speech Lang Pathol. 2010;19:34-50.

10. Kohnert K. Language disorders in bilingual children and adults. 2nd ed. San Diego: Plural Publishing; 2013.

11. Gershkoff-Stowe L, Thelen E. U-shaped changes in behavior: a dynamic systems perspective. J Cogn Dev. 2004;5:11-36.

12. Goldstein B, Washington P. An initial investigation of phonological patterns in 4-year-old typically developing Spanish-English bilingual children. Lang Speech Hear Serv Sch. 2001;32:153-64.

13. Gildersleeve-Neumann CE, Kester ES, Davis BL, Peña E. English speech sound development in preschool-aged children from bilingual English-Spanish environments. Lang Speech Hear Serv Sch. 2008;39:314-28.

14. Goldstein, B. (2000, June). Phonological disorders in bilingual (Spanish-English) children. Seminar presented at the 2000 Child Phonology Conference. Cedar Falls, IA; 2000.

15. Gildersleeve-Neumann CE, Peña EP, Davis BL, Kester ES. Effects on L1 during early acquisition of L2: speech changes in Spanish at first English contact. Biling Lang Cogn. 2009;12:259-72. Results from this study indicated cross-linguistic effects of English on the children's first language, Spanish, although cross-linguistic transfer did not affect all properties of the phonology equally.

16. Gildersleeve-Neumann C, Goldstein BA. Cross-linguistic generalization in the treatment of two sequential Spanish-English bilingual children with speech sound disorders. Int J Speech Lang Pathol. 2014. doi:10.3109/17549507.2014.898093. Results from this treatment study indicated that children's speech improved in both languages for treated and some non-treated error patterns. Treatment in both languages had an overall positive effect on the speech of sequential bilingual children.

17. Dodd B, So LKH, Wei L. Symptoms of disorder without impairment: the written and spoken errors of bilinguals. In: Dodd B, Campbell R, Worrall L, editors. Evaluating theories of language: evidence from disordered communication. London: Whurr; 1996. p. 119-36.

18. Grech H, Dodd B. Phonological acquisition in Malta: a bilingual learning context. Int J Biling. 2008;12:155-71.

19. Kehoe M, Trujillo C, Lleó C. Bilingual phonological acquisition: an analysis of syllable structure and VOT. In: Cantone KF, Hinzelin M-O, editors. Proceedings of the colloquium on structure, acquisition and change of grammars: phonological and syntactic aspects, vol. 27. Arbeiten zur Mehrsprachigkeit: Universität Hamburg; 2001. p. 38-54.

20. Lleó C, Kuchenbrandt I, Kehoe M, Trujillo C. Syllable final consonants in Spanish and German monolingual and bilingual acquisition. In: Müller N, editor. (In) vulnerable domains in multilingualism. Amsterdam, Philadelphia: John Benjamins; 2003. p. 191-220.

21. Goldstein B, Fabiano L, Washington P. Phonological skills in predominantly English, predominantly Spanish, and Spanish-English bilingual children. Lang Speech Hear Serv Sch. 2005;36:201-18. 
22. Gildersleeve-Neumann CE, Wright KE. English phonological acquisition in 3- to 5-year-old children learning Russian and English. Lang Speech Hear Serv Sch. 2010;41:429-44.

23. Burrows L, Goldstein B. Whole word measures in bilingual children with speech sound disorders. Clin Linguist Phon. 2010;24: 357-68.

24. Goldstein, B., Bunta, F., Lange, J., Burrows, L., Pont, S., \& Bennett, J. Interdependence in the phonological systems of bilingual children with speech sound disorders. Seminar presented at the convention of the American Speech-Language-Hearing Association, Chicago, IL: 2008

25. Wolfram W, Schilling-Estes N. American English: dialects and variation. Oxford: Blackwell; 1998.

26. Leather J, James A. Second language speech. In: Ritchie W, Bhatia T, editors. Handbook of second language acquisition. San Diego: Academic Press; 1996. p. 269-316.

27. Schnitzer M, Krasinski E. The development of segmental phonological production in a bilingual child: a contrasting second case. $\mathrm{J}$ Child Lang. 1996;23:547-71.

28. Law N, So L. The relationship of phonological development and language dominance in bilingual Cantonese-Putonghua children. Int J Biling. 2006;10:405-27.

29. Goldstein B, Bunta F, Lange J, Rodriguez J, Burrows L. The effects of measures of language experience and language ability on segmental accuracy in bilingual children. Am J Speech-Lang Pathol. 2010;19:238-47.

30. Shriberg LD, Lohmeier HL, Strand EA, Jakielski KJ. Encoding, memory, and transcoding deficits in childhood apraxia of speech. Clin Linguist Phon. 2012;26:445-82.

31. Shriberg LD, Austin D, Lewis BA, McSweeny JL, Wilson DL. The speech disorders classification system (SDCS): extensions and lifespan reference data. J Speech Lang Hear Res. 1997;40:723-40.

32. Shriberg LD, Austin D, Lewis BA, McSweeny JL, Wilson DL. The percentage of consonants correct (PCC) metric: extensions and reliability data. J Speech Lang Hear Res. 1997;40:708-22.
33. Dale PS. The validity of parent report measure of vocabulary and syntax at 24 months. J Speech Hear Res. 1991;34:565-71.

34. Feldman HM, Dollaghan CA, Campbell TF, Kurs-Lasky M, Janosky JE, Paradise JL. Measurement properties of the MacArthur communicative development inventories at ages one and two years. Child Dev. 2000;71:310-22.

35. Guiberson M, Rodriguez BL. Measurement properties and classification accuracy of two Spanish parent surveys of language development for preschool-age children. Am J Speech Lang Pathol. 2010;3:225-37.

36. Restrepo MA. Identifiers of predominantly Spanish-speaking children with language impairment. J Speech Lang Hear Res. 1998;6: 1398-411.

37. Stertzbach JB, Gildersleeve-Neumann CE. Parent report as a speech screening tool in Spanish-speaking children. Technical session presented at the annual meeting of the American speech-languagehearing association. CA: San Diego; 2006.

38. Gildersleeve-Neumann CE, Appenzeller RT. 30-minute technical session presented at the annual ASHA Conference. Orlando: FL; 2014. Speech sound disorders in Spanish-English bilingual children: characteristics and role of parent surveys for identification.

39. Glaspey A, Stoel-Gammon C. A dynamic approach to phonological assessment. Adv Speech-Lang Pathol. 2007;9:286-96.

40. Holm A, Ozanne A, Dodd B. Efficacy of intervention for a bilingual child making articulation and phonological errors. Int J Biling. 1997;1:55-69.

41. Holm A, Dodd B. Comparison of cross-language generalisation following speech therapy. Folia Phoniatr Logop. 2001;53:166-72.

42. Ray J. Treating phonological disorders in a multilingual child: a case study. Am J Speech Lang Pathol. 2002;11:305-15.

43. Ebert KD, Kohnert T. Common factors in speech-language treatment: an exploratory study of effective clinicians. J Commun Disord. 2010;43:133-47. 